\title{
Microscopic and macroscopic volume conduction in skeletal muscle tissue, applied to simulation of single-fibre action potentials
}

\author{
B. A. Albers W. L. C. Rutten \\ W. Wallinga-de Jonge, H. B. K. Boom \\ University of Twente, Department of Electrical Engineering/Biomedical Engineering Division, PO Box 217, \\ 7500 AE Enschede, The Netherlands.
}

\begin{abstract}
Extracellular action potentials of a single active muscle fibre in a surrounding of passive muscle tissue were calculated, using a microscopic volume conductor model which accounts for the travelling aspect of the source, the structure of skeletal muscle tissue and the electrical properties at the level of single muscle fibres. Owing to the capacitive properties of the muscle fibre membranes this inhomogeneous model is frequency dependent. The results of these calculations were compared with results obtained with a macroscopic homogeneous, frequency-independent version of the model. Close to the excited fibre the extracellular action potentials of both descriptions differ in their sensitivity to variations in the intra-and extracellular conductivities. It is demonstrated that within about $300 \mu \mathrm{m}$ the muscle fibre membrane influences the amplitude as well as the timing of the peaks of the extracellular action potential. Both volume conduction descriptions result in almost the same single-fibre action potential at large radial distances from the excited fibre. These model results are of primary interest for the quantitative interpretation of single-fibre electromyograms recorded close to an active fibre, as for example in clinical fibre-density measurements.
\end{abstract}

Keywords-Muscle fibre membrane, Single fibre action potential, Skeletal muscle, Tissue structures, Volume conduction

Med. \& Biol. Eng. \& Comput, 1988, 26, 605-610

\section{Introduction}

IN HOMOGENEOUS volume conductor models of skeletal muscle tissue (used for example by ROSENFALCK, 1969; NANDEDKAR and STÅLBERG, 1983) the tissue has no structure. It is considered as a bulk material with anisotropic conductivities $\sigma_{z}$ (in the longitudinal fibre direction) and $\sigma_{r}$ (radial). Usually, $\sigma_{z}$ and $\sigma_{r}$ are taken real-valued. Albers et al. (1986) introduced a microscopic, frequency-dependent volume conductor model to study the relationship between SFAP and tissue structure. This network model accounted explicitly for the fibrous structure of skeletal muscle tissue. It was used for the calculation of the transfer function between a moving current source and the resulting extracellular potential (ALBERS et al., 1988). It was shown that, close to the source, the transfer function is significantly different from the transfer function calculated with a homogeneous, frequency-independent volume conductor model. This is a direct effect of the fibrous structure of the tissue in the inhomogeneous, capacitive model. In the

Correspondence should be addressed to Dr Rutten.

Bert Albers is now at Cordis Europa, PO Box 38, 9300 AA Roden, The Netherlands.

First received 28th April 1987 and in final form 17th May 1988

(C) IFMBE: 1988 present paper the effects of this fundamental difference between both transfers are analysed with respect to the SFAP in the time domain. In contrast to the previous article in which the homogeneous case was calculated analytically, we followed a different strategy: now the homogeneous model is obtained by proper substitutions and omissions inside the inhomogeneous network model itself.

\section{Simulation model}

For clarity, we reformulate briefly the basic elements of the modelling, as described more extensively in earlier papers (ALBERS et al., 1986; 1988).

In its basic concept the model consists of a large number of nodes. Each node is identified uniquely by a crosssectional index $c\left(-N_{c} \leqslant c \leqslant N_{c}\right.$, with $2 * N_{c}+1$ the total number of cross-sections), and an index $n$, defining the radial position within the cross-section. The longitudinal separation is given by the discretisation length $L$ (Fig. 1).

The extracellular response $V_{n}(\omega)$ in node $n$ of cross section $c=0$ is given by

$$
V_{n}(\omega)=H_{n}(\omega) I(\omega)
$$

with $\omega=2 \pi f$, where $f$ is the frequency of the impressed current. $I(\omega)$ is the total current injected by the active fibre 


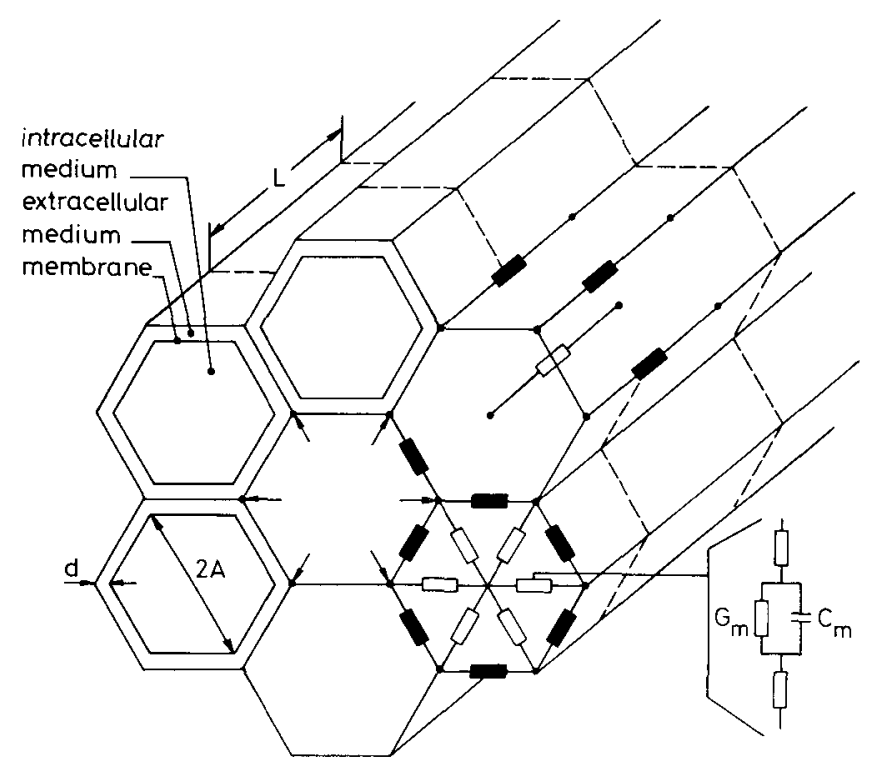

Fig. 1 Model structure, in which the muscle tissue is represented by an assembly of identical fibres with a hexagonal transverse surface. In the longitudinal direction the network is cross-sectioned in 'slices' with thickness $L$. The transformation into an electrical network is also illustrated in this figure. Current is injected only in one cross-section, in the six extracellular nodes of the central fibre, indicated by arrows

in cross section $c=0$. The transfer function $H_{n}(\omega)$ is given by

$$
H_{n}(\omega)=Z_{0, n}(\omega)+2\left\{\sum_{c=1}^{c=N_{c}} Z_{c, n}(\omega) \cos \left(\omega c \frac{L}{U}\right)\right\}
$$

Impedances $Z_{c, n}(\omega)$ describe the amplitude and phase relationship between a potential at point $(c, n)$ and a current injected at $c=0$. These impedances are calculated numerically, using an electrical network representation of skeletal muscle tissue. The spatial distribution of the current source represents a part of the active fibre membrane with length $L$ (Fig. 1). The transfer function (eqn. 2) gives a full description of volume conduction in the case of a moving current source (conduction velocity $U$ is included in eqn. 2); however, it does not depend on other temporal properties of the source. After multiplication with the Fourier transform of a proper membrane current and inverse Fourier transformation of this product, the result is the SFAP in the time domain. The membrane current $I_{m}(t)$ of a membrane part with length $L$ reads

$$
I_{m}(t)=\frac{\pi A^{2} L \sigma_{i}}{U^{2}} \cdot \frac{\partial^{2} v_{i}(t)}{\partial t^{2}}
$$

The intracellular action potential $v_{i}(t)$ is approximated by the expression (after RosenfalcK, 1969):

$$
v_{i}(t)=\alpha t^{3} \exp (-\beta t)-\gamma
$$

with $\alpha, \beta$ and $\gamma$ constants determining the shape of the action potential. A detailed description of the network model and the solution of the impedances $Z_{c, n}(\omega)$ is given by Albers et al. (1986). Parameters of the model are listed in Table 1.

In a macroscopic approximation skeletal muscle tissue is considered as a homogeneous anisotropic volume conductor with conductivities $\sigma_{r}$ and $\sigma_{z}$ in the directions normal and parallel to the fibre direction. To use the network for the simulation of the homogeneous case one has to translate the network parameters $\sigma_{i}, \sigma_{e}, p, A, G_{m}$ and $C_{m}$ into $\sigma_{z}$ and $\sigma_{r}$.

The relationship between the homogeneous parameters $\sigma_{z}$ and $\sigma_{r}$ and the microscopic parameters of the inhomogeneous network model is derived analytically for the model structure of Fig. 1 by GIELEN et al. (1986):

$$
\begin{aligned}
& \sigma_{z}=(1-p) \sigma_{e}+p \sigma_{i} \\
& \sigma_{r}=\frac{1-p}{1+p} \sigma_{e}+\frac{\sqrt{3}}{2} A\left(G_{m}+j \omega C_{m}\right)
\end{aligned}
$$

with $p$ the intracellular volume fraction.

In the frequency range, covered by EMG signals and using realistic parameter values, the term $(\sqrt{3} / 2) A\left(G_{m}\right.$ $\left.+j \omega C_{m}\right) \equiv 1 / Z_{m}$ contributes only a small part to $\sigma_{r}$. Therefore this term will be neglected, so both $\sigma_{r}$ and $\sigma_{z}$ become frequency independent.

\section{Results}

Fig. 2 shows the intracellular action potential used in the simulations. Parameters $\alpha, \beta$ and $\gamma$ are given in the legend. This intracellular action potential as well as all extracellular action potentials are calculated with a resolution of $20 \mu \mathrm{s}$. The sensitivity of the SFAP obtained with both volume conductor models, to variations in the intra- and extracellular conductivity is examined by using three different parameter sets, listed in Table 1 . The choice of the microscopic parameters results in realistic values of $\sigma_{Z}$ and $\sigma_{r}$, as reported for example by EPSTEIN and FosTER (1983) and ZHENG et al. (1984).

Fig. 3 presents results for the three parameter sets of Table 1. The SFAP at a radial distance of $53 \mu \mathrm{m}$ from the centre of the active fibre is shown, plus the radial decline of the parameters $A_{t t}$ and $A_{t t} / \sigma_{i} . A_{t t}$ is the sum of the amplitudes of the first and second phases of the SFAP. Because the amplitude of the membrane current $I_{m}(t)$ is proportional to $\sigma_{i}$ (see eqn. 3), $A_{t t} / \sigma_{i}$ is a better parameter for a source-independent comparison than $A_{t t}$ alone.

Fig. 3 shows that the amplitude of the SFAP in the

Table 1 Parameter values used in the simulations

\begin{tabular}{clcccc}
\hline Symbol & \multicolumn{1}{c}{ Parameter } & $A$ & $B$ & $C$ & unit \\
\hline$\sigma_{i}$ & intracellular conductivity & 0.750 & 0.450 & 0.750 & $(\Omega \mathrm{m})^{-1}$ \\
$\sigma_{e}$ & extracellular conductivity & 2.500 & 2.500 & 1.800 & $(\Omega \mathrm{m})^{-1}$ \\
$\sigma_{z}$ & parallel homogeneous conductivity & 0.925 & 0.655 & 0.855 & $(\Omega \mathrm{m})^{-1}$ \\
$\sigma_{r}$ & transverse homogeneous conductivity & 0.131 & 0.131 & 0.095 & $(\Omega \mathrm{m})^{-1}$ \\
$K^{2}$ & anisotropy ratio $K^{2}=\sigma_{z} \sigma_{r}$ & 7.040 & 4.984 & 9.038 & -
\end{tabular}

For all parameter sets :

$A$ fibre radius

$d \quad$ extracellular layer thickness

$p \quad$ intracellular volume fraction

$C_{m}$ membrane capacitance per unit surface area

$G_{m}$ membrane conductance per unit of surface area
$25 \mu \mathrm{m}$

$1.35 \mu \mathrm{m}$

$0 \cdot 9$

$0.01 \mathrm{~F} \mathrm{~m}^{-2}$ $1 \Omega^{-1} \mathrm{~m}^{-2}$

The parameters are within the range indicated by COLE and CUR TIS (1950) 


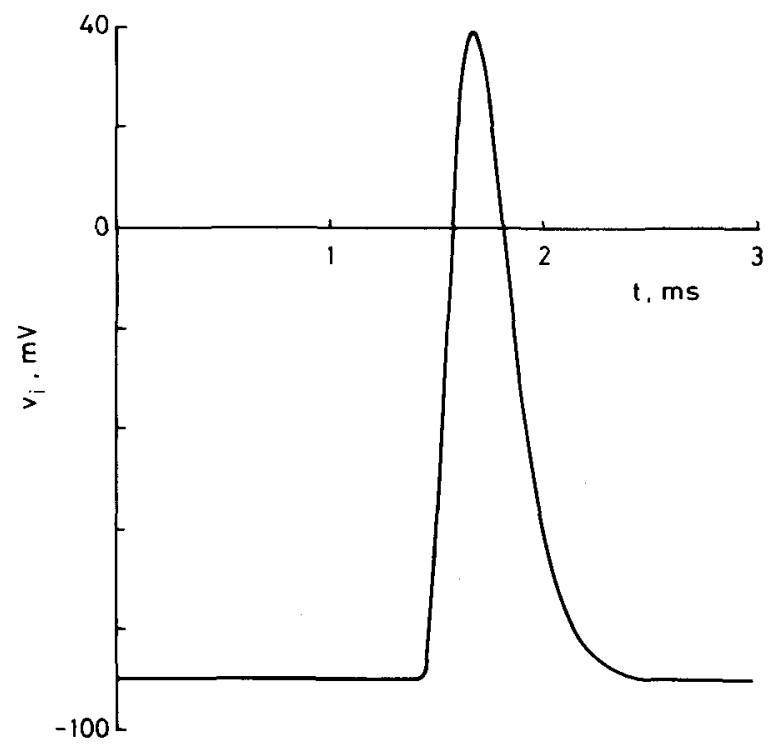

Fig. 2 Intracellular action potential, used in the simulations to calculate the membrane current. The action potential was calculated with reln. 4, using the following parameter values: $\alpha=166 \times 10^{9} \mathrm{~V} \mathrm{~s}^{-3} ; \beta=12 \times 10^{3} \mathrm{~s}^{-1} ; \gamma=$ $0.09 \mathrm{~V}$
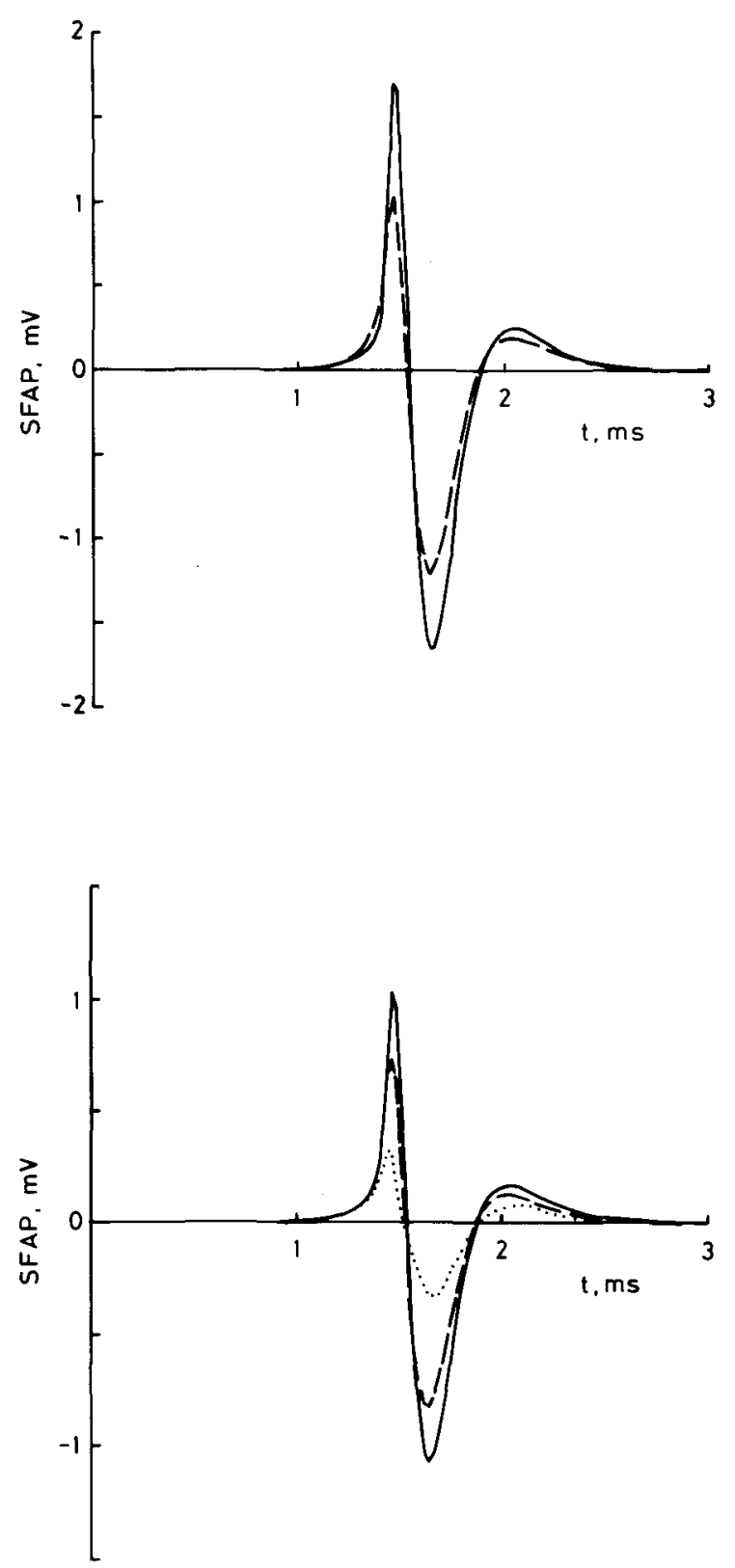

Fig. 3 Caption overleaf inhomogeneous medium is higher than in the homogeneous medium. For small radial distances the SFAP amplitude calculated with the inhomogeneous medium exceeds the corresponding homogeneous result by about 50 per cent. For larger distances $(>300 \mu \mathrm{m})$ the SFAP amplitudes are about equal in both models.

The SFAPs in the two models differ in their sensitivity to variations of the intra- and extracellular conductivities, as illustrated in Fig. 3. With respect to the parameter $A_{t t} / \sigma_{i}$ the homogeneous model shows a slightly larger sensitivity to a decrease in $\sigma_{i}$ than the inhomogeneous model. On the other hand, the inhomogeneous model is much more sensitive to a decrease in $\sigma_{e}$ than the homogeneous model.

This difference in sensitivity to $\sigma_{i}$ and $\sigma_{e}$ reflects the different way in which current is being redistributed in the homogeneous and inhomogeneous volume conductor, respectively. In the inhomogeneous medium and close to the source, current is being conducted almost exclusively through the extracellular medium and complete redistribution is reached at a large distance from the source, which implies a high sensitivity to the extracellular conductivity. In the homogeneous model redistribution is not influenced by the structure (AlBERS et al., 1986).
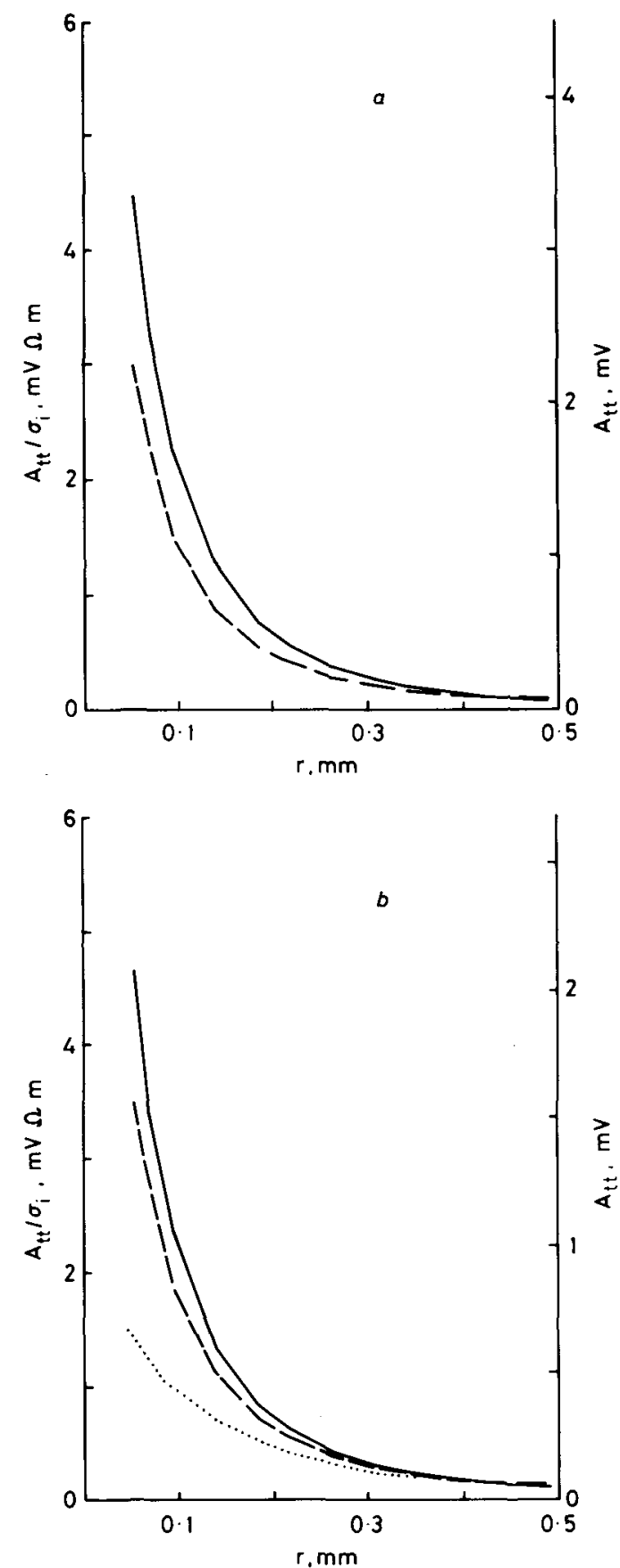

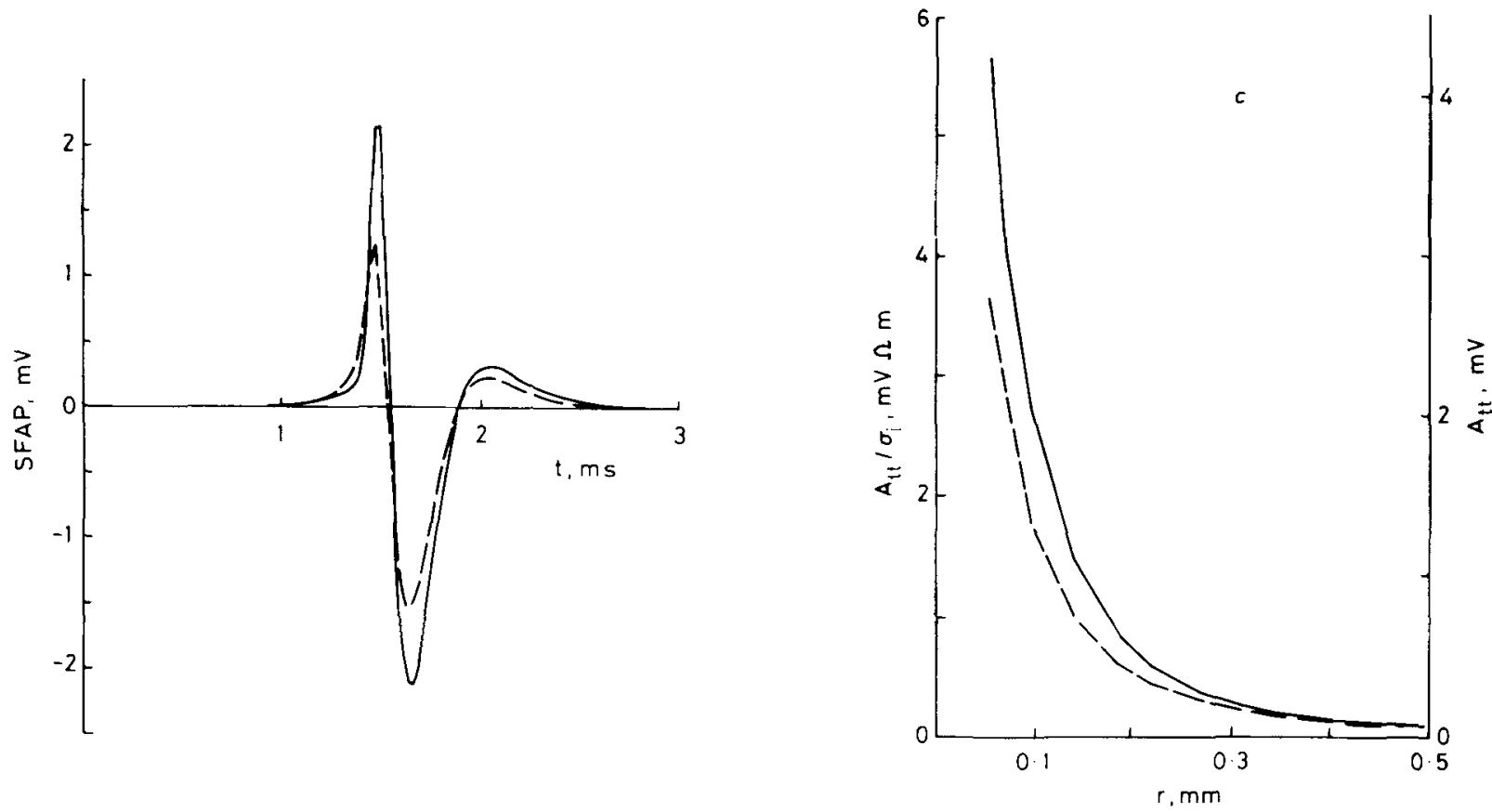

Fig. $3 S F A P S$ calculated at a radial distance of $53 \mu \mathrm{m}$ from the centre of the excited fibre and the radial decline of the parameters $A_{t t} / \sigma_{i}$ and $A_{u} .(a),(b)$ and $(c)$ represent results for parameter sets $A, B$ and $C$, respectively. Solid lines: inhomogeneous medium; broken lines: homogeneous medium. Dotted line in $(b)$ : inhomogeneous medium with extra $G_{e}$ and $C_{e}$ components in the membrane part of the model (see Discussion). The conduction velocity is $3 \mathrm{~m} \mathrm{~s}^{-1}$

Fig. 4 shows the latency shift of the position of the first positive peak of the SFAP with increasing radial distances relative to the latency of this peak at a distance of $53 \mu \mathrm{m}$. The inhomogeneous results show a small positive time shift for parameter set A only, while for sets B and C the position of the first peak is not influenced by the increase of radial distance. On the contrary, the inhomogeneous results show a significant negative shift for all three parameter sets.

As described in eqn. 2, the transfer function $H_{n}(\omega)$ and consequently the SFAP are summations of a number of contributions. Consequently, the ultimate SFAP shape is determined by the amplitude and phase of these individual contributions. Because in the homogeneous description volume conduction is purely resistive, phase shifts cannot be due to capacitive effects, so in this case the phase of each contribution is only determined by the propagation of the source. As a result the first peak of the action potential shows a negative time shift if the radial distance increases, as illustrated in Fig. 4. In the inhomogeneous description, the fibre membrane influences the redistribution of current, affecting the amplitude as well as the phase of the individual contributions to the SFAP. As a result the timing of the first peak of the action potentials is almost unchanged.

The influence of the fibre membrane was studied in more detail by an additional simulation using the inhomogeneous model, but without considering complex valued impedances. So, the complex value of the membrane impedance $Z_{m}=(\sqrt{3} / 2) A\left(G_{m}+j \omega C_{m}\right)^{-1}$ was replaced by its modulus $\left|Z_{m}\right|=(\sqrt{3} / 2) A\left(G_{m}^{2}+\omega^{2} C_{m}^{2}\right)^{-1 / 2}$

In Fig. 5 results obtained before and after this substitution are compared with SFAPs calculated with the homogeneous medium at radial distances of $70 \mu \mathrm{m}$ and $468 \mu \mathrm{m}$ using parameter set $\mathrm{B}$ of Table 1 . After the substitution $Z_{m}=\left|Z_{m}\right|$ the homogeneous and inhomogeneous descriptions result in almost the same SFAP at large radial distance. First, this implies that in this situation the relationship between parameters of homogeneous and inhomogeneous description (eqns. 5 and 6) are correct. Secondly, it indicates that the differences between results of both descriptions at large distance, observed before the substitution, are only caused by phase effects of the fibre membrane. Because the SFAP close to the excited fibre is also slightly influenced by the substitution $Z_{m}=\left|Z_{m}\right|$, the general conclusion is that close to the excited fibre the
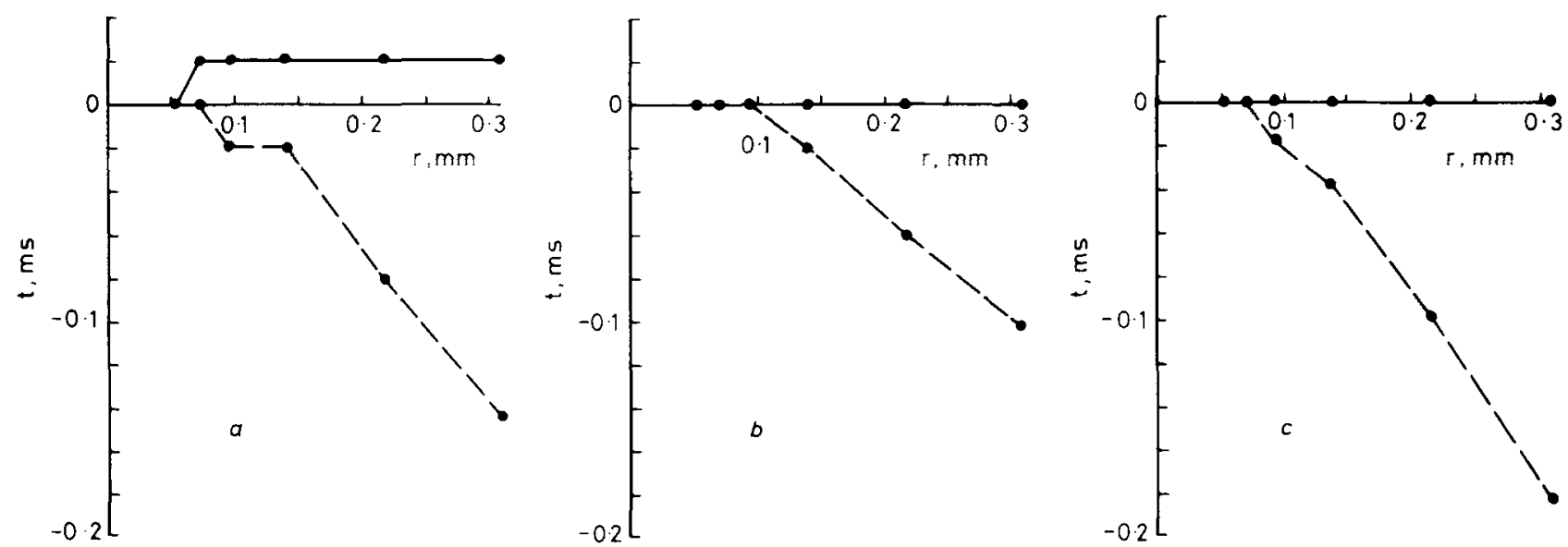

Fig. 4 Time shift in the position of the first positive peak of the SFAP as a function of the radial distance from the excited fibre. Negative values indicate a shift to the left on the time axis. $(a),(b)$ and (c) represent results for parameter sets $A, B$ and $C$, respectively. Solid lines: inhomogeneous medium; broken lines: homogeneous medium 

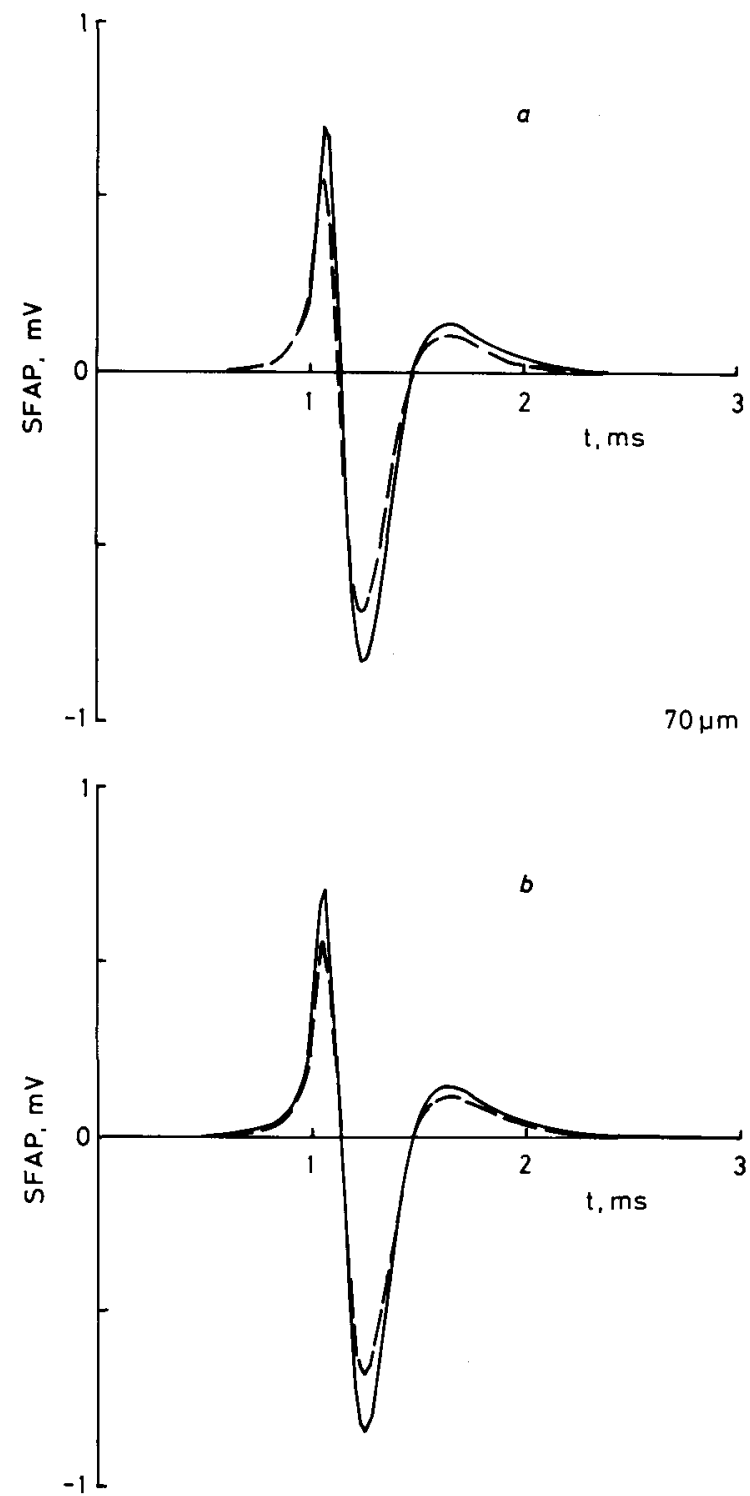
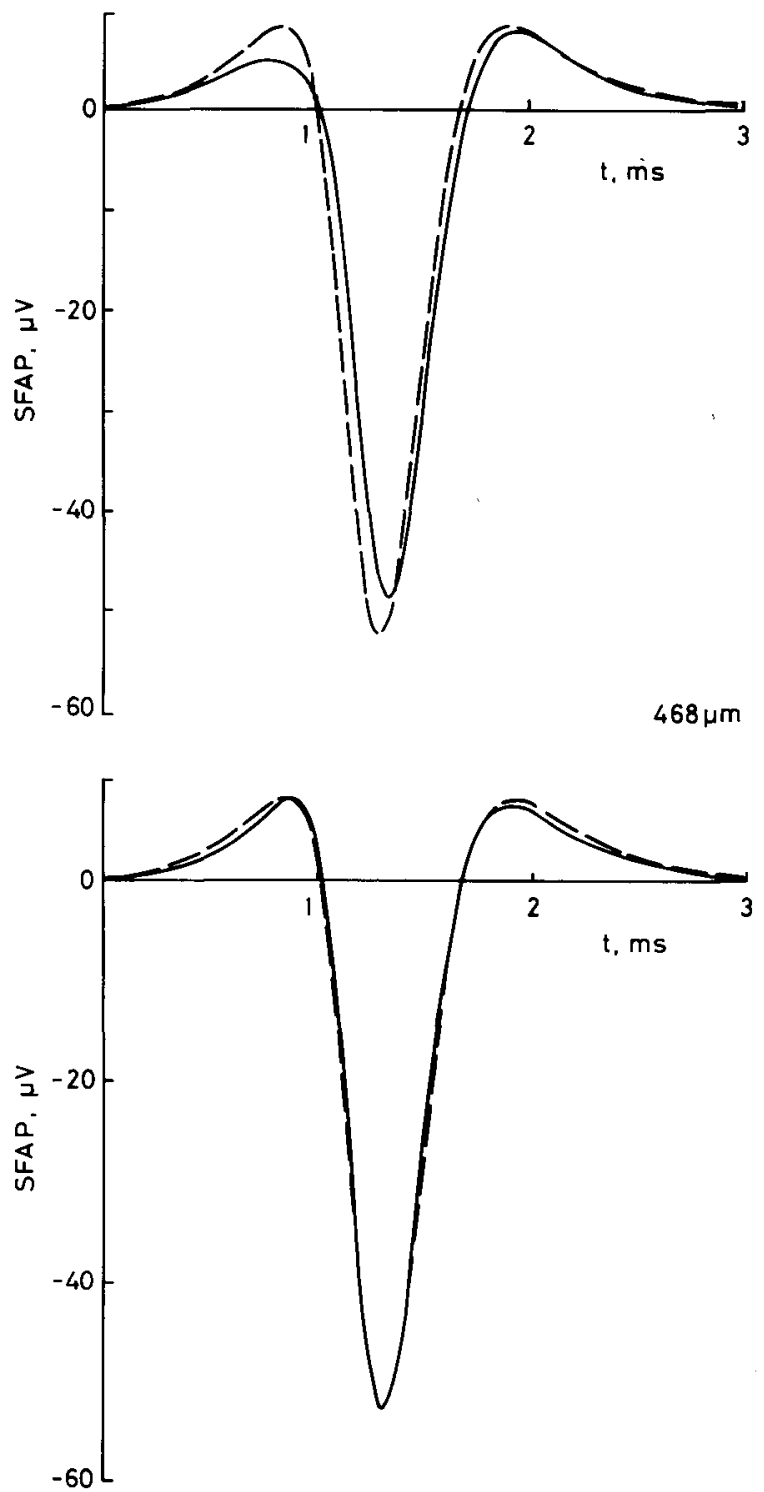

Fig. 5 Effect of the substitution $Z_{m}=\left|Z_{m}\right|$, using parameters set $B$ and a conduction velocity of $3 \mathrm{~m} \mathrm{~s}^{-1}$. Homogeneous and inhomogeneous results are indicated with broken and solid lines, respectively. Radial distances from the centre of the excited fibre are $70 \mu \mathrm{m}$ and $468 \mu \mathrm{m}$. (a) SFAPs before the substitution; (b) SFAPs after the substitution

amplitude effect as well as the phase effect of the membrane is observed, whereas at large distance only the phase effect is noticed.

\section{Discussion}

At large radial distances the inhomogeneous result for $Z_{m}=\left|Z_{m}\right|$ closely resembles the SFAP calculated for the homogeneous medium (Fig. 5). This implies that in this situation the relationship between parameters of homogeneous and inhomogeneous description (eqns. 5 and 6) are correct.

In all the results, the SFAP amplitude obtained close to the excited fibre in the inhomogeneous medium exceeds that in the homogeneous medium. It has been reported that SFAP amplitudes, calculated with the usual homogeneous approach, are too low with respect to recorded potentials. Very high SFAP amplitudes as recorded for example by EKSTEDT (1964) are never obtained with a homogeneous volume conductor model. The results of the present paper suggest that a microscopic description is to be preferred above variation of macroscopic parameters in the homogeneous description (as suggested for instance by PatTle, 1971 and NANDEDKar and Stålberg 1983). The present results also imply the limited value of SFAP recordings obtained from isolated fibres immersed in a homogeneous solution (for instance EKsTEDT, 1964, GYDIKov et al., 1986).

The results of Fig. 3 show that the SFAP amplitude has declined to $200 \mu \mathrm{V}$ at a distance of $300 \mu \mathrm{m}$ for parameter sets $A$ and $C$ and at $260 \mu \mathrm{m}$ for parameter set B. These values are in good accordance with experimental data reported by GATH and STÅLBERG (1979). These authors defined the pick-up radius of a single-fibre electrode as the distance at which the action potential amplitude has declined to $200 \mu \mathrm{V}$. An alternative, relative definition of the pickup radius is the distance at which the SFAP amplitude is one-tenth of the highest observed value (PoLlak, 1971). Fig. 3 shows the advantage of the absolute definition of Gath and Stålberg above the latter. In the definition of Gath and Stallberg the pick-up radius is almost insensitive to the extracellular parameter $\sigma_{e}$ (see Figs. $3 a$ and $3 c$ ).

This independence upon extracellular conductivity implies that fibre packing may vary throughout the tissue without affecting too much the pickup radius (provided however that the fibre radius A does not vary). Clinical fibre density measurements may therefore benefit from the choice of an absolute criterion for the pickup radius. However, it has to be noted that variations in fibre radius and conduction velocity can shift the pickup radius considerably (eqn. 3). 
The contribution $Z_{m}^{-1}=(\sqrt{3} / 2) A\left(G_{m}+j \omega C_{m}\right)$ (see eqn. 6) represents one of the simplest models for the surface membrane of the muscle fibre, i.e. the parallel combination of a membrane capacitance $C_{m}$ and a membrane conductance $G_{m}$. More refined models should reflect the presence of the tubular system (T-system) as well. This has been tried by FALK and FATT (1964) and by many others more recently (EISENBERG, 1983) all based on measurements of the linear electrical behaviour of muscle fibres in several species. FALK and FATT (1964) added a series $G_{e} C_{e}$ combination in parallel to the parallel $G_{m} C_{m}$ combination to account for the tubular system. With this addition they could successfully describe their experimental, complex impedance data. For the frog they used the values $C_{e}=$ $0.041 \mathrm{~F} \mathrm{~m}^{-2}, G_{e}=30 \Omega^{-1} \mathrm{~m}^{-2}, G_{m}=3 \Omega^{-1} \mathrm{~m}^{-2}$ and $C_{m}=0.026 \mathrm{~F} \mathrm{~m}^{-2}$.

In the rat, values for $C_{e}$ and $G_{e}$ are not known from the experimental literature. The values for $G_{m}$ and $C_{m}$ in the rat are $1 \Omega^{-1} \mathrm{~m}^{-2}$ and $0.01 \mathrm{~F} \mathrm{~m}^{-2}$, respectively (see Table $1)$; i.e. a factor of about three smaller than in frog. We shall apply the same scaling factor to scale down $C_{e}$ and $G_{e}$. With these choices $\left(C_{e}=0.014 \mathrm{~F} \mathrm{~m}^{-2}, G_{e}=10 \Omega^{-1}\right.$ $\mathrm{m}^{-2}, G_{m}=1 \Omega^{-1} \mathrm{~m}^{-2}$ and $C_{m}=0.010 \mathrm{~F} \mathrm{~m}^{-2}$ ) network simulation gives results of which the two dotted curves in Fig. $3 b$ are examples. Compared with the solid curves in the same figure an attenuation of $V_{u}$ (of at most 70 per cent for $r=53 \mu \mathrm{m}$ ) has taken place, while the SFAP shape is unchanged. There is almost no attenuation beyond about $300 \mu \mathrm{m}$.

These results indicate the urgent need for accurate membrane parameter data to calculate SFAP amplitudes at a microscopic scale. As we have assumed certain $C_{e}$ and $G_{e}$ values for the rat, because of the lack of experimental values, the dotted results in Fig. $3 b$ only have a tentative meaning. The strong reduction of the SFAP amplitudes upon introduction of $G_{e}$ and $C_{e}$ enhances the discrepancy between the experimental (high) (EKSTEDT, 1964; GATH and STÅLBERG 1979) and simulated (low) amplitudes.

\section{References}

Albers, B. A., Rutten, W. L. C., Wallinga-de Jonge, W. and BoOM, H. B. K. (1986) A model study on the influence of structure and membrane capacitance on volume conduction in skeletal muscle tissue. IEEE Trans., BME-33, 681-689.

Albers, B. A., Rutten, W. L. C., Wallinga-de Jonge, W. and BooM, H. B. K. (1988) Frequency domain modelling of volume conduction of single muscle fiber action potentials. IEEE Trans., BME-35, 328-333.

Cole, K. S. and CuRTIS, H. J. (1950). Bio-electricity. electric physiology. In Medical physics, vol. 2. Glasser, O. (ed), Year Book, Chicago, Illinois, 82-89.

EISENBERG, R. S. (1983) Impedance measurement of the electrical structure of skeletal muscle. In Handbook of physiology. Peachy, L. E. (Ed.), American Physiological Society, Bethesda, Maryland.

EKSTEDT, J. (1964) Human single muscle fiber action potentials. Acta Physiol. Scand., 61, Suppl. 226, 1-96.

EPStein, B. R. and Foster, K. R. (1983) Anisotropy in the dielectric properties of skeletal muscle. Med.\& Biol. Eng. \& Comput., 21, 51-55.

FALK, G. and FATT, P. (1964) Linear electrical properties of stimulated muscle fibres observed with intracellular electrode. Proc. R. Soc. London, B160, 69-123.

GATH, I. and STAlberg, E. (1979) Measurements of the uptake area of small size electromyographic electrodes. IEEE Trans., BME-26, 374-376.

Gielen, F. L. H., Cruts, H. E. P., Albers, B. A., Boon, K. L., Wallinga-DE JoNGE, W. and BoOM, H. B. K. (1986) Model of electrical conductivity of skeletal muscle based on tissue structure. Med.\& Biol. Eng. \& Comput., 24, 3440.
Gydikov, A., Gerilovsky, L., Radicheva, N. and Trayanova, $N$. (1986) Influence of the muscle fibre end geometry on the extracellular potentials. Biol. Cybern., 54, 1-8.

NANDEDKAR, S. D. and STÅlbERG, E. (1983) Simulation of single muscle fibre action potentials. Med.\& Biol. Eng. \& Comput., 21, $158-165$.

Pattle, R. E. (1971) The external action potential of a nerve or muscle fibre in an extended medium. Phys. In Med. \& Biol., 16, 673-685.

Pollax, V. (1971) The waveshape of action potentials recorded with different types of electromyographic needles. Med. \& Biol. Eng., 9, 657-664.

ROSENFALCK, P. (1969) Intra- and extracellular potential fields of active nerve and muscle fibres. A physicomathematical analysis of different models. Akademisk Forlag, Copenhagen.

Zheng, E., Shao, S. and Webster, J. G. (1984) Impedance of skeletal muscle from $1 \mathrm{~Hz}$ to $1 \mathrm{MHz}$. IEEE Trans., BME-31, $477-481$.

\section{Authors' biographies}

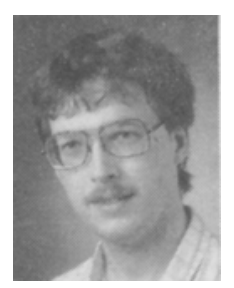

Bert A. Albers was born in Hengelo, The Netherlands, in 1958. He received his M.Sc. degree in Physics from Twente University of Technology in 1983 and joined the Biomedical Engineering Division, Department of Electrical Engineering, University of Twente His research interests were electrophysiology and volume conduction in skeletal muscle tissue. He received the Ph.D. degree in Biomedical Engineering in 1987 and in the same year joined Cordis Europa NV, where he is involved with clinical evaluation of angiographic products.

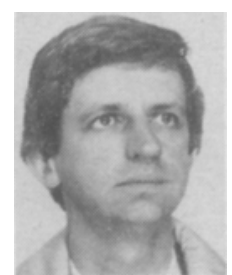

Wim L. C. Rutten was born in The Hague, The Netherlands, in 1950 . He received the M.Sc. degree in Experimental Physics in 1974 and a Ph.D. in Physics in 1979 from the University of Leiden. He began working on solid-state magnetism research at the Kamerlingh Onnes Laboratory, Leiden in 1972 and in experimental and clinical audiology at the University Hospital, Leiden from 1979 to 1985 . Since 1985 he has been a senior staff member of the Biomedical Engineering Group, University of Twente. His main current research interests are volume conduction in muscle, selective neural stimulation, and surface EMG.

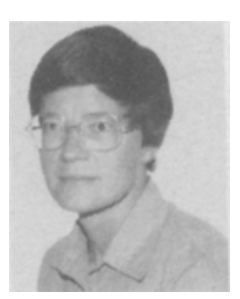

Willemien Wallinga-de Jonge was born in Veendam, The Netherlands, in 1945. She received the M.Sc. degree in Biology from the University of Groningen in 1968 and her Ph.D. in Biomedical Engineering from Twente University of Technology, Enschede, in 1980. She is a senior staff member of the Biomedical Engineering Group, Department of Electrical Engineering of Twente University of Technology. Her research activities there involve the electrical activity of muscle in general and the intracellular and extracellular single fibre activity in particular.

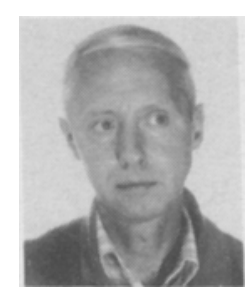

Herman B. K. Boom was trained as a medical physicist at the University of Utrecht, where he received his Ph.D. in 1971. He joined the Departments of Medical Physics and Medical Physiology where he was engaged in research in the field of cardiac mechanics and taught physiology and biophysics. Since 1976 he has occupied the Chair of Medical Electronics in the Electrical Engineering Department of Twente University of Technology. His research interests are cardiovascular system dynamics, bioelectricity and rehabilitation technology 